\title{
Mutant COQ2 in Multiple-System Atrophy
}

TO THE EDITOR: Tsuji and colleagues (July 18 issue) ${ }^{1}$ report that variants in the gene encoding coenzyme $\mathrm{Q}_{2}$ (COQ2) increase the risk of multiple-system atrophy. They observed homozygous COQ2 variants encoding the substitutions M78V and V343A in a consanguineous Japanese family with multiple-system atrophy subtype $\mathrm{P}$ and noted an association between V343A and sporadic multiple-system atrophy (minor-allele frequency [MAF], $4.8 \%$ of cases vs. $1.6 \%$ of controls; odds ratio, 3.05; 95\% confidence interval, 1.65 to 5.85). However, the authors erroneously labeled human $\mathrm{COQ} 2$ variability from the fourth ATG start codon in exon 1, which encodes the smallest protein isoform and does not functionally complement the yeast coq2-null mutant. ${ }^{2}$ On the basis of the National Center for Biotechnology Information (NCBI) Reference Sequence (NM_015697.7), M78V should be labeled COQ2 c.382A $\rightarrow$ G (p.M128V) and V343A should be labeled c.1178T $\rightarrow$ C (p.V393A). We sequenced COQ2 in 299 Korean persons with multiple-system atrophy and 365 unaffected Korean persons and observed heterozygous COQ2 c.320G $\rightarrow \mathrm{C}$ (encoding p.S107T) and c.382A $\rightarrow \mathrm{T}$ (encoding p.M128R) in 2 patients with sporadic multiple-system atrophy; COQ2 c.1178T $\rightarrow$ C (p.V393A) was not associated with multiple-system atrophy (MAF, $2.7 \%$ of cases vs. $2.6 \%$ of controls).

It is a challenge to reconcile recessive linkage of homozygous COQ2 mutations in familial multiple-system atrophy with a heterozygous, presumably dominant-negative association in sporadic multiple-system atrophy. Respectfully, we suggest that Tsuji and colleagues reconsider whether variations in COQ2 represent a risk factor for multiple-system atrophy. Genomic multiplications of the SNCA 6.4-Mb locus telomeric to COQ2 have previously been implicated in parkinsonism and multiple-system atrophy ${ }^{3}$; copy number analysis of linked loci, or genomewide analysis, should be considered.

Beom S. Jeon, M.D.

Seoul National University

Seoul, South Korea

brain@snu.ac.kr
Matt J. Farrer, Ph.D.

Stephanie F. Bortnick, B.Sc.

University of British Columbia

Vancouver, BC, Canada

for the Korean Canadian Alliance on

Parkinson's Disease and Related Disorders

No potential conflict of interest relevant to this letter was reported.

1. The Multiple-System Atrophy Research Collaboration. Mutations in COQ2 in familial and sporadic multiple-system atrophy. N Engl J Med 2013;369:233-44.

2. López-Martín JM, Salviati L, Trevisson E, et al. Missense mutation of the COQ2 gene causes defects of bioenergetics and de novo pyrimidine synthesis. Hum Mol Genet 2007;16:1091-7.

3. Ross OA, Braithwaite AT, Skipper LM, et al. Genomic investigation of alpha-synuclein multiplication and parkinsonism. Ann Neurol 2008;63:743-50.

DOI: 10.1056/NEJMc1311763

TO THE EDITOR: Tsuji and colleagues identified one compound heterozygous mutation and one homozygous mutation in COQ2 in two multiplex Japanese families with multiple-system atrophy. The gene encodes parahydroxybenzoate-polyprenyl transferase, an enzyme essential to the biosynthesis of coenzyme $\mathrm{Q}_{10}$ (COQ10). The authors also described heterozygous sequence changes in COQ2 in sporadic cases of multiple-system atrophy. We directly assessed the association of these putative pathogenic variants with disease in a large European cohort of 788 persons with multiple-system atrophy, 1900 persons with Parkinson's disease and 600 unaffected persons. We found two of the described variants in the COQ2 gene, S347C (referred to by Tsuji and colleagues as S297C) and V393A (referred to by Tsuji and colleagues as V343A), in 1 patient each in the cohort of patients with Parkinson's disease and found the P157S variant (referred to by Tsuji and colleagues as P107S) in 1 unaffected person. We did not observe any of the variants described by Tsuji and colleagues in the European cohort of patients with multiple-system atrophy, suggesting that these variants are population-specific.

Manu Sharma, Ph.D.

Hertie Institute for Clinical Brain Research

Tübingen, Germany

manu.sharma@uni-tuebingen.de 
Gregor Wenning, M.D.

Innsbruck Medical University

Innsbruck, Austria

Rejko Krüger, M.D.

German Center for Neurodegenerative Diseases

Tübingen, Germany

for the European Multiple-System Atrophy

Study Group (EMSA-SG)

Members of the EMSA-SG consortium are listed in the Supplementary Appendix, available with the full text of this letter at NEJM.org.

Drs. Sharma, Wenning, and Krüger contributed equally to this letter.

No potential conflict of interest relevant to this letter was reported.

DOI: 10.1056/NEJMc1311763

TO THE EDITOR: The cause of multiple-system atrophy is obscure. There exist reports of association between the disease and variants in SNCA, the gene encoding $\alpha$-synuclein, ${ }^{1-3}$ and Tsuji and colleagues report an association between $\mathrm{COQ} 2$ variants in Japanese patients and familial and sporadic clinically diagnosed multiple-system atrophy. Their study was based on linkage analysis in families with multiple-system atrophy and on genome sequencing, through which homozygous and compound heterozygous $\mathrm{COQ} 2$ mutants were detected in two families. Decreased growth rates in COQ2 variants identified in multiple-system atrophy were detected in a yeast complementation assay, a finding consistent with the view that the association with disease was caused by loss of function in the protein encoded by COQ2. In a multicenter collaboration funded by the Medical Research Council of the United Kingdom, the Wellcome Trust, and the Multiple-System Atrophy Trust, we sequenced coding regions and flanking introns of COQ2 (ENST00000311469, CCDS47090.2, NM_015697.7) in more than 300 patients with neuropathologically confirmed multiple-system atrophy (see the Supplementary Appendix, available with the full text of this letter at NEJM.org) and 262 controls, all of European descent.

We identified a COQ2 nonsense variant that was more common in controls than in persons with multiple-system atrophy (R22X, 24 vs. 9 alleles; $\mathrm{P}<0.002)$ and two other variants (rs6818847, p.V66L; and rs6535454, p.D298D), which were also more common in controls (see Table S1 in the Supplementary Appendix for provenance of samples). We failed to detect the variant V393A (referred to by Tsuji and colleagues as V343A) that was reported to be associated with multiplesystem atrophy in Japanese patients. (See Table S2 in the Supplementary Appendix for the results of coding of COQ2 variants.) Our data do not support an association between loss-of-function COQ2 variants and increased risk of multiplesystem atrophy in Europeans.

Lucia V. Schottlaender, M.D.

Henry Houlden, M.D., Ph.D.

UCL Institute of Neurology

London, United Kingdom

h.houlden@ucl.ac.uk

for the Multiple-System Atrophy (MSA) Brain

Bank Collaboration

Members of the MSA Brain Bank Collaboration are listed in the Supplementary Appendix.

No potential conflict of interest relevant to this letter was reported.

1. Scholz SW, Houlden H, Schulte C, et al. SNCA variants are associated with increased risk for multiple system atrophy. Ann Neurol 2009;65:610-4.

2. Ross OA, Vilariño-Güell C, Wszolek ZK, Farrer MJ, Dickson DW. Reply to: SNCA variants are associated with increased risk of multiple system atrophy. Ann Neurol 2010;67:414-5.

3. Al-Chalabi A, Dürr A, Wood NW, et al. Genetic variants of the alpha-synuclein gene SNCA are associated with multiple system atrophy. PLoS One 2009;4(9):e7114.

DOI: 10.1056/NEJMc1311763

TO THE EDITOR: Tsuji and colleagues describe the association of functionally impaired variants of COQ2 with parkinsonism or cerebellar ataxia in multiple-system atrophy, but they did not explain that cerebellar ataxia with variable involvement of the central nervous system is strongly associated with both primary and secondary COQ10 deficiency, with onset often in adulthood. ${ }^{1}$ In fact, although reports suggest that COQ2 mutations can cause isolated glucocorticoid-resistant nephropathy, to our knowledge, the first patient reported to harbor a homozygous COQ2 mutation had visual dysfunction attributed to rod-cone retinopathy and encephalopathy, with indications on magnetic resonance imaging of diffuse cerebral and cerebellar atrophy as well as bilateral lesions in the cingulate cortex and subcortical area. $^{2,3}$ Ophthalmologic problems are also often observed in patients with primary COQ10 defi- 
ciencies. ${ }^{1}$ It is noteworthy, then, that the only proven pathogenic mutation described by Tsuji and colleagues (p.M78V-V343A) occurred in two siblings from a consanguineous family with multiple-system atrophy, retinitis pigmentosa, and low levels of COQ10 in the cerebellum, levels that are similar to those previously reported in the muscle tissue of patients with primary COQ10 deficiency. ${ }^{1}$

Catarina M. Quinzii, M.D.

Michio Hirano, M.D.

Salvatore DiMauro, M.D.

Columbia University Medical Center

New York, NY

mh29@cumc.columbia.edu

No potential conflict of interest relevant to this letter was reported.

1. Emmanuele V, López LC, Berardo A, et al. Heterogeneity of coenzyme Q10 deficiency: patient study and literature review. Arch Neurol 2012;69:978-83.

2. Salviati L, Sacconi S, Murer L, et al. Infantile encephalomyopathy and nephropathy with CoQ10 deficiency: a CoQ10-responsive condition. Neurology 2005;65:606-8.

3. Quinzii C, Naini A, Salviati L, et al. A mutation in parahydroxybenzoate-polyprenyl transferase (COQ2) causes primary coenzyme Q10 deficiency. Am J Hum Genet 2006;78:345-9.

DOI: 10.1056/NEJMc1311763

THE AUTHORS REPLY: We thank Jeon and colleagues for bringing the matter of variant annotation to our attention and have corrected the annotation in the article such that amino acids are numbered with respect to the first ATG codon (rather than the fourth ATG codon) in exon 1 of COQ2 (NCBI Reference Sequence, NM_015697.7). ${ }^{1}$ We focused our study entirely on COQ2 variants occurring distal to the fourth ATG codon because the protein isoform starting at the fourth ATG codon has been adopted as the canonical sequence of the $\mathrm{COQ} 2$ protein in the UniProt database (Q96H96 isoform-1, www.uniprot.org/uniprot/ Q96H96) and a strong cross-species conservation is observed across this isoform. In contrast, the cross-species conservation is weak across the amino-acid sequences located upstream of the methionine encoded by the fourth ATG codon (see the Supplementary Appendix, available with the full text of this letter at NEJM.org). Moreover, RNA-sequencing data available in public databases, including ENCODE (http://genome.ucsc.edu/ ENCODE) and DBTSS (http://dbtss.hgc.jp), indicate that the majority of the transcripts in human tissues start near the third or fourth ATG codon. Further investigations would be required to determine the transcription start site and the translation initiation codon for the appropriate annotation of COQ2.

Schottlaender and Houlden found that rs112033303 (R22X) is more common in controls than in cases. Because rs112033303 lies upstream of the fourth ATG codon, we did not include it in our study. We agree that their data do not support the association of this variant with susceptibility to multiple-system atrophy.

Jeon and colleagues, Sharma and colleagues, and Schottlaender and Houlden tested for association between COQ2 variants and multiple-system atrophy in their case-control series and observed none. Studies using larger sample sizes and involving tests of the effect on $\mathrm{COQ} 2$ function may be required to detect an association.

We reported the association of the allele frequency of V393A with multiple-system atrophy in a Japanese series $(4.8 \%$ in patients with multiple-system atrophy vs. 1.6 to $2.2 \%$ in controls). Jeon and colleagues also found V393A in Korean controls, but they did not find an association $(2.7 \%$ vs. $2.6 \%)$. In our analysis, V393A was strongly associated with the cerebellar subtype of multiple-system atrophy ( $6.0 \%$ vs. 1.6 to $2.2 \%$ ), but not with the parkinsonian subtype (2.4\% vs. 1.6 to $2.2 \%$ ); the statistical power to detect an association probably depends on the prevalence of each subtype in the sample tested.

Jeon and colleagues raise the possibility of multiplications of SNCA, located near COQ2, as a cause of multiple-system atrophy, but this prospect seems unlikely in Family 1; our data support a recombination event between COQ2 and SNCA in an affected member of Family 1.

Noting that cerebellar ataxia is the most common clinical presentation associated with primary and secondary COQ10 deficiencies, Quinzii and colleagues suggest that the multiple-system atrophy phenotype could be part of a broad clinical spectrum of COQ10 deficiency. It would also be important to investigate $\alpha$-synuclein neuropathology in primary COQ10 deficiency.

\footnotetext{
Jun Mitsui, M.D., Ph.D.

Shoji Tsuji, M.D., Ph.D.

University of Tokyo

Tokyo, Japan

tsuji@m.u-tokyo.ac.jp
} 
Since publication of their article, the authors report no further potential conflict of interest.

1. López-Martín JM, Salviati L, Trevisson E, et al. Missense mu- tation of the COQ2 gene causes defects of bioenergetics and de novo pyrimidine synthesis. Hum Mol Genet 2007;16:1091-7.

\section{Albumin Replacement in Severe Sepsis or Septic Shock}

TO THE EDITOR: The Albumin Italian Outcome Sepsis study conducted by Caironi et al. (April 10 issue $)^{1}$ is the third large-scale, randomized trial to compare albumin with crystalloids in adult patients with severe sepsis. The first such trial was the Saline versus Albumin Fluid Evaluation study. ${ }^{2}$ In addition, the Early Albumin Resuscitation during Septic Shock study has been completed and its mortality results published. ${ }^{3}$

In all three trials, mortality was lower among patients receiving albumin, and the respective relative risks coincided closely, ranging from 0.87 to 0.94 (Fig. 1). Although the effect did not attain statistical significance in any of the individual trials, the pooled relative risk in all three trials is $0.92(95 \%$ confidence interval [CI], 0.84 to $1.00 ; \mathrm{P}=0.046$ ), indicating a significant reduction in mortality associated with albumin use among adults with severe sepsis. This result supports the conclusion of a previous metaanalysis involving predominantly small trials with different control fluids and patient populations. ${ }^{4}$ The result is also consistent with the observed reduction in mortality associated with albumin use among patients with spontaneous bacterial peritonitis, a disease that shares im- portant pathophysiological features with severe sepsis. ${ }^{5}$

Christian J. Wiedermann, M.D.

Central Hospital of Bolzano

Bolzano, Italy

christian.wiedermann@asbz.it

Michael Joannidis, M.D.

Medical University of Innsbruck

Innsbruck, Austria

Dr. Wiedermann reports receiving lecture fees from CSL Behring, Baxter, and the Plasma Protein Therapeutics Association; and Dr. Joannidis, lecture fees from Baxter, Fresenius, Gambro, Orion Pharma, CLS Behring, and B. Braun Melsungen. No other potential conflict of interest relevant to this letter was reported.

1. Caironi P, Tognoni G, Masson S, et al. Albumin replacement in patients with severe sepsis or septic shock. $\mathrm{N}$ Engl J Med 2014;370:1412-21.

2. The SAFE Study Investigators. Impact of albumin compared to saline on organ function and mortality of patients with severe sepsis. Intensive Care Med 2011;37:86-96.

3. Charpentier J, Mira J-P. Efficacy and tolerance of hyperoncotic albumin administration in septic shock patients: the EARSS study. Intensive Care Med 2011;37:Suppl 1:S115.

4. Delaney AP, Dan A, McCaffrey J, Finfer S. The role of albumin as a resuscitation fluid for patients with sepsis: a systematic review and meta-analysis. Crit Care Med 2011;39:386-91.

5. Salerno F, Navickis RJ, Wilkes MM. Albumin infusion improves outcomes of patients with spontaneous bacterial peritonitis: a meta-analysis of randomized trials. Clin Gastroenterol Hepatol 2013;11:123-30.

DOI: 10.1056/NEJMc1405675

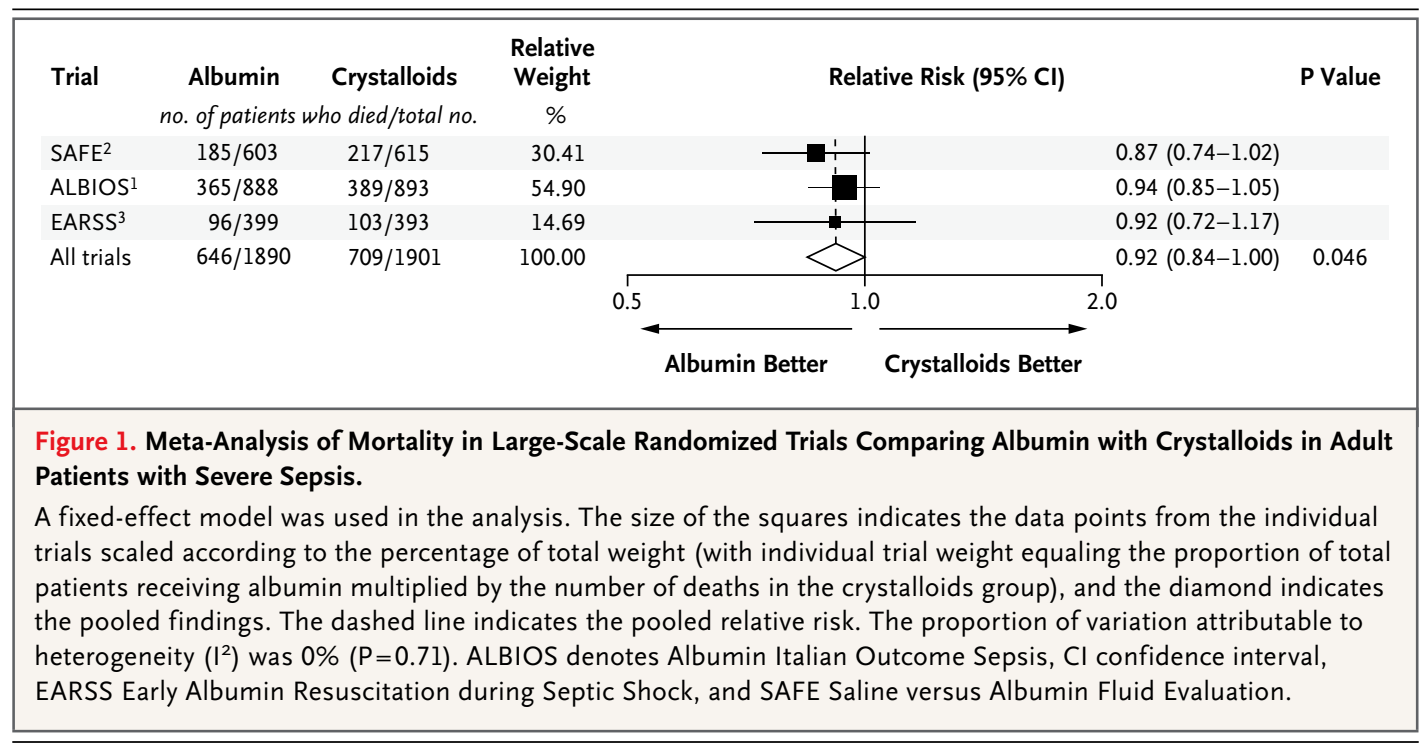

